\title{
GESTÃO DO CONHECIMENTO EM UMA COOPERATIVA MÉDICA DO NORTE/NORDESTE BRASILEIRO
}

Lydia Maria Pinto Brito lydiampbrito@yahoo.com.br Doutor em Educação pela Universidade Federal do Ceará (UFC). Professora Titular do Programa de Pós Graduação em Administração da Universidade Potiguar UnP/RN.

Sérgio Ricardo Vasconcelos Santos sergiorvasconcelos@ hotmail.com

Mestre em Administração de empresas pela Universidade Potiguar (UnP). Professor do Programa de Pós-Graduação Laureate International Universities.

Walid Abbas El-Aouar walidbranco@gmail.com Doutor em Administração pela Universidade Federal do Rio Grande do Norte (UFRN). Professor titular e Coordenador do Mestrado Profissional em Administração da Universidade Potiguar (UnP).

\footnotetext{
Ahiram Brunni Cartaxo de Castro brunnicastro@hotmail.com Doutorando em Administração pela Universidade Potiguar (UnP). Administrador no Instituto Federal de Educação, Ciência e Tecnologia do Rio Grande do Norte (IFRN).
}

\section{RESUMO}

O objetivo do estudo foi analisar o estágio da Gestão do Conhecimento (GC) na percepção de gestores e analistas de uma Cooperativa Médica do Norte/Nordeste Brasileiro. O modelo teórico utilizado foi o de Bukowitz e Williams (2002) com definição de variáveis em Brito, Oliveira e Castro (2012). Tratou-se de uma pesquisa qualitativa com modo de investigação no estudo de caso e enfoque descritivo. Os sujeitos da pesquisa foram os 24 gestores e 50 analistas da cooperativa estudada. Os dados foram coletados por meio de questionários e tratados conforme o algoritmo do modelo em planilhas eletrônicas. Os resultados sinalizaram valores superiores aos propostos por Bukowtiz e Williams (2002), demonstrando-se pela implantação da GC tanto no nível tático quanto no estratégico na organização estudada. Sendo assim, é possível observar que a organização propicia condições facilitadoras para a criação, o reconhecimento do conhecimento tácito, a disseminação e incorporação do conhecimento às tecnologias e aos produtos; entretanto, a organização não conseguiu consolidar a fase de avaliação, que é o momento em que se define o conhecimento necessário para sua manutenção no futuro.

Palavras-chave: Gestão do Conhecimento. Cooperativas Médicas. Norte e Nordeste Brasileiro.

\section{INTRODUÇÃO}

Vive-se em um momento histórico de inovação em produtos e serviços, impulsionado pelo uso do conhecimento em tecnologia da informação e da comunicação, o qual contribui para uma maior dinâmica no mercado e resulta no aumento da concorrência. Observa-se assim que as organizações passaram a se comportar como mercados do conhecimento.

O conhecimento tem sido visto como um dos mais importantes recursos das organizações por ser capaz de tornar as ações, no plano interorganizacional (parcerias, pesquisa), intraorganizacional (estrutura organizacional, tecnologias, políticas de gestão), grupal (liderança) e individual (confiança e colaboração), mais inteligentes, efetivas e facilmente compartilhadas e documentadas (LEE, 
2017).

Nesse contexto, o modelo de Gestão do Conhecimento se constitui em um conjunto integrado de ações para identificar, capturar, gerenciar e disseminar todo o ativo intelectual de informações de uma organização (PINHEIRO et al., 2016).

Entretanto, para a consolidação desse modelo nas organizações, uma série de desafios deve ser superada, entre eles: a criação de uma cultura organizacional de aprendizagem e de compartilhamento do conhecimento (SENGE, 2012; TANG, 2017); a definição de papéis de gestão do conhecimento (trabalhadores do conhecimento - diretores de gestão do conhecimento, analistas da informação, ciberoticários, mentores, entre outros) viabilizadores da mudança (BRITO; OLIVEIRA; CASTRO, 2016); o planejamento de espaços organizacionais; a criação de times multidisciplinares de aprendizagem; a consecução de práticas de gestão do conhecimento como comunidades virtuais (LARA et al., 2017); e melhores práticas de GC (PETROVA et al., 2017), entre outros.

Discutir a Gestão do Conhecimento em uma cooperativa médica em uma ambiência de alta competitividade, como é a dos planos de saúde, pode contribuir para despertar o interesse por sua implementação, consolidação e aplicabilidade, por meio da construção de estratégias de melhorias dos serviços prestados aos cooperados e clientes internos e externos e resultados empresariais e sociais. Além disso, a ciência médica evolui, e é necessário que a promoção da saúde esteja em sintonia com as melhores práticas da academia, do mercado e da sociedade.

Nesse sentido, a questão de pesquisa que emerge é: qual o estágio da Gestão do Conhecimento na percepção de gestores e analistas de uma cooperativa médica do Norte e Nordeste brasileiro que implantaram esse modelo?

O objetivo geral do estudo foi analisar o estágio da Gestão do Conhecimento na percepção de gestores e analistas em uma Cooperativa Médica no Norte e Nordeste Brasileiro e os objetivos específicos foram: descrever os fatores determinantes do processo tático para obter, utilizar, aprender e contribuir com o conhecimento da organização; e verificar, por meio do processo estratégico, como o conhecimento é avaliado, construído e descartado.

Em seguida, tem-se a abordagem teórica do trabalho, o detalhamento dos procedimentos metodológicos adotados, a apresentação e discussão dos resultados, e as considerações provisórias.

\section{ABORDAGEM TEÓRICA}

Segundo Osinski (2015), a Gestão do Conhecimento ainda é um tema relativamente novo, uma vez que as publicações a respeito começaram a se intensificar somente a partir de 2009. Dessa forma, embora a discussão teórica dos clássicos tenha iniciado na década de 1990, só recentemente as práticas recebem, gradativamente, a atenção de gestores e pesquisadores de diversas áreas, principalmente das Ciências da Informação e da Administração. Sendo, portanto, um tema que está em constante evolução, pois se busca complementar lacunas e desenvolver construtos, em um processo de desenvolvimento, de ampliação do campo de conhecimento - tipo evolutivo (BOXENBAUM; ROULEAU, 2011; NOGUEIRA; ODELIUS, 2015), e que não para de ser revisado, pois existe espaço de consolidação para os conceitos e pressupostos de aprendizagem em organizações, principalmente para o século XXI, considerado como o século das redes sociais e da informação democratizada.

\subsection{GESTÃO DO CONHECIMENTO}

Em meio às crescentes mudanças e dificuldades que as organizações vêm enfrentando, em um cenário de mercado cada vez mais competitivo, a Gestão do Conhecimento tem mostrado excelentes fontes de trabalho antes esquecidas. Nas organizações, fatores como capital intelectual, cultura organizacional, utilização de recursos intangíveis e de novas tecnologias estão aumentando a capacidade de se trabalhar com a crescente complexidade do 
mercado, tornando a Gestão do Conhecimento um importante fator estratégico para a sustentabilidade organizacional.

A Gestão do Conhecimento sempre existiu quando, por exemplo, as empresas familiares transferiam o conhecimento a seus descendentes em atividades específicas, bem como os navegadores ensinavam a seus aprendizes, fazendo assim que se evoluísse o conhecimento no desempenho de suas funções. Contudo, não era um processo consciente e formal que se constituía em um modelo teórico, pois somente a partir da década de 1990 é que o conhecimento atingiu maiores proporções de desenvolvimento, e a Gestão do Conhecimento foi-se concebendo como um modelo para a gestão das organizações. Com o advento das tecnologias de informação, principalmente da internet, a disseminação do conhecimento entre organizações e indivíduos tem crescido grandemente, pois não existem mais limites geográficos para a interação entre pessoas, organizações e nações (SHEHZAD; KHAN, 2013; KIANTO et al., 2014; CHON; BESHARATI, 2014).

Duas proposições tornaram-se muito importantes no pensamento administrativo contemporâneo e dizem respeito ao conhecimento, ou melhor, a sua gestão. A Gestão do Conhecimento é essencial para as organizações atingirem vantagem competitiva. Além de ser uma preocupação central, deve-se tornar uma competência essencial.

Por Gestão do Conhecimento entende-se a destinação de recursos e a doação de técnicas gerenciais na geração, disseminação e administração de conhecimentos estratégicos, com o objetivo de gerar resultados econômicos para a empresa e benefícios para os stakeholders, assim entendidos todos os públicos que têm interesses diretos ou indiretos na organização (TERRA, 2005; VIRTANEN, 2013; MASSINGHAM, 2014; TZORTZAKI; MIHIOTIS, 2014).

Bukowitz e Williams (2002) identificam que a Gestão do Conhecimento é um processo pelo qual a organização gera riqueza, a partir da construção de seu capital intelectual. Brito
(2005) complementa afirmando que as organizações baseadas em conhecimento possuem atividades centrais, como concepção, desenvolvimento de políticas e estratégias.

Para Davenport e Prusak (1998); Gonzalez e Martins (2014), as organizações comportam-se como mercados do conhecimento, existindo compradores (as pessoas que buscam o conhecimento para solucionar algum problema), vendedores (aquelas que possuem reputação no mercado interno por terem conhecimento substancial sobre o processo ou assunto) e as intermediárias (pessoas que realizam as conexões entre compradores e vendedores).

O modelo de avaliação da Gestão do Conhecimento proposto Bukowitz e Williams (2002) segue dois cursos de atividades estruturadas que ocorrem simultaneamente dentro das organizações, que são: o processo tático - que se refere à utilização de conhecimento no dia-a-dia para responder às demandas ou às oportunidades do mercado - e o processo estratégico, focado mais em longo prazo para combinar o intelectual com as exigências estratégicas. 


\section{Processo Tático}

\begin{tabular}{|c|l|}
\hline \multicolumn{2}{|c|}{ Processo Tático } \\
\hline Obtenção & $\begin{array}{l}\text { Etapa na qual as pessoas procuram informações para resolver problemas, tomar } \\
\text { decisões ou criar produtos e serviços novos; }\end{array}$ \\
\hline Utilização & $\begin{array}{l}\text { Fase em que se utiliza a informação para criar soluções inovadoras, estimulando a } \\
\text { criatividade dentro da organização; }\end{array}$ \\
\hline Aprendizagem & Estágio caracterizado por compreendero modo como se adquirem novos conhecimentos; \\
\hline Contribuição & $\begin{array}{l}\text { Fase relativa à participação do indivíduo junto à organização, quando concede seu } \\
\text { conhecimento para compor o conhecimento organizacional. }\end{array}$ \\
\hline Avaliação & $\begin{array}{l}\text { Momento em que a organização define o conhecimento necessário para sua missão e } \\
\text { mapeia o capital intelectual, projetando as necessidades futuras de conhecimento. }\end{array}$ \\
\hline Construção & $\begin{array}{l}\text { Etapa relativa à elaboração e manutenção do conhecimento que agrega valor ao } \\
\text { negócio da organização. }\end{array}$ \\
\hline Descarte & $\begin{array}{l}\text { Fase relativa ao abandono do conhecimento que não agrega mais valor ao negócio ou } \\
\text { se distancia do core business da organização. }\end{array}$ \\
\hline
\end{tabular}

Quadro 1 - Processos da Gestão do Conhecimento

Fonte: adaptado de Galvão (2012).

Os dois processos são integrados e buscam a manutenção das organizações no mercado por meio do capital intelectual que reside nas pessoas/trabalhadores.

\subsection{CAPITAL INTELECTUAL}

Stewart (1998) afirma que o capital intelectual é, na verdade, a nova vantagem competitiva das empresas, em uma clara alusão aos trabalhos de Porter (1990). Ele é também bastante enfático ao definir o capital intelectual como a soma dos conhecimentos de todos em uma organização, fato que propicia à empresa uma vantagem competitiva. Em seu trabalho, Stewart (1998) recomenda que desprezem as distinções arbitrárias entre dados, informações, conhecimentos e sabedoria. Segundo o autor, esta é uma discussão interminável e apenas se deve reconhecer a importância do conhecimento tácito. $\mathrm{O}$ autor relata que se pode encontrar o capital intelectual em três lugares: pessoas, estruturas e clientes.

Sveiby (1998), complementando, tipifica as organizações do conhecimento com base em duas características precípuas, entre outras: a primeira delas é a que se refere à qualificação do corpo de trabalhadores, responsáveis pela conversão de informação em conhecimento, utilizando-se de suas próprias competências, com ou sem auxílio de fornecedores da informação ou de conhecimento especializado. Uma segunda característica dessas organizações é o fato de que elas possuem poucos ativos tangíveis ao passo que seus ativos intangíveis representam o verdadeiro valor.

O capital intelectual também é definido por Bukowitz e Williams (2002, p. 18), como "qualquer coisa valorizada pela organização que esteja contida nas pessoas, ou seja, derivada de processos, de sistemas e da cultura organizacional." Ainda nesse contexto, Bukowitz e Williams (2002) ressaltam que é importante compreender que o capital intelectual não é propriedade da organização, não podendo, portanto, estar sob seu controle direto.

Na visão de Terra (2005); Virtanen (2013); Jasimuddin e Zhang (2014), a questão da mensuração do capital intelectual sofreu um grande impulso em meados dos anos de 1990, em um trabalho pioneiro desenvolvido na empresa sueca de serviços financeiros, Skandia. Essa experiência dividiu esse capital em dois grandes grupos: o capital humano e o capital estrutural. Portanto, quando as organizações inovam, elas não processam simplesmente a organização de fora para dentro, visando solucionar problemas 
existentes e adaptar-se ao ambiente em mudança. Elas realmente criam novos conhecimentos e informações, de dentro para fora, visando redefinir tanto os problemas quanto as soluções, por meio das habilidades das pessoas de recriar seus ambientes e se diferenciar dos concorrentes. É no exercício da conversão do capital intelectual que se originam as organizações de aprendizagem (CASTRO, 2011; WAHYUNINGSIH; ASTUTI; MUSADIEQ, 2013; SOMALINGAN; SHANTHAKUMARI, 2013; MEIHAMI; MEIHAMI, 2014; MASSARO; DUMAY; GARLATTI, 2015).

A construção do conhecimento dependerá também da capacidade da organização de gerir conflitos cognitivos que são induzidos principalmente por discrepâncias entre a estrutura conceptual cognitiva de alguém por um lado, e a estrutura conceitual apresentada pelo artefato digital por outro. As pessoas podem resolver este conflito, principalmente, alterando a sua estrutura de conhecimento individual pela assimilação interna e alojamento (KIMMERLE; CRESS; HELD, 2010; SOMALINGAN; SHANTHAKUMARI, 2013), ou pela internalização, socialização, externalização e combinação (NONAKA; TAKEUCHI, 1997).

A emergência de um conflito cognitivo acontece em decorrência da diferença entre a ideia de alguém de como realizar uma determinada tarefa e o procedimento apresentado em um padrão compartilhado. Entretanto, esse fato pode incentivar o repensar do método e resultar no desenvolvimento de um novo procedimento para executar a tarefa, o que pode levar à melhoria da construção do conhecimento, das tecnologias de apoio e da gestão organizacional para combinar processos de aprendizagem individual e coletiva. Como exemplo, as ferramentas de software podem ser particularmente úteis para fins de construção de conhecimento se elas fornecerem oportunidades para manipular e influenciar, de forma compartilhada, artefatos digitais que tenham a capacidade de induzir e gerir conflitos cognitivos. Os futuros desenvolvimentos de software e tecnologias de comunicação para fins de construção de conhecimento e gestão do conhecimento serão suporte estratégico fundamental para a evolução desta prática administrativa (KIMMERLE; CRESS; HELD, 2010; KIM et al. 2014; WONG et al., 2015).

Os estudos de Hautala (2011) sinalizam que a proximidade social e conceitual e a confiança são fundamentais para a implantação da Gestão do Conhecimento organizacional. A autora observa que, quando o conhecimento criado inclui conceitos abstratos e discussões filosóficas, são necessárias habilidades de linguagem mais profundas que possam permitir a compreensão dos valores culturais por trás da linguagem e seu uso análogo, que as características individuais afetam a interpretação e a continuidade do discurso, e que os fatores estruturais, como administração, financiamento e colaboração são fundamentais.

\section{PROCEDIMENTOS METODOLÓ- GICOS}

Trata-se de uma pesquisa qualitativa com modo de investigação no estudo de caso e enfoque descritivo (GODOY, 1995).

Para Merriam (1998), o interesse da pesquisa qualitativa está na compreensão de interpretações sobre um ponto particular no tempo e em um contexto. Nesse sentido, coaduna com o propósito desta pesquisa,

\begin{abstract}
O estudo de caso tem se tornado a estratégia preferida quando os pesquisadores procuram responder às questões "como" e "por que" certos fenômenos ocorrem, quando há pouca possibilidade de controle sobre os eventos estudados e quando o foco de interesse é sobre fenômenos atuais, que só poderão ser analisados dentro de algum contexto de vida real (GODOY, 1995, p. 26).
\end{abstract}

O estudo de caso possibilita ainda uma descrição intensiva e a análise de um fenômeno com um indivíduo, um grupo, uma instituição ou uma comunidade (MERRIAM, 1998).

Quanto à validade interna e a confiabilidade desta pesquisa, tem-se que o estudo pas- 
sou por avaliação com pares, com professores doutores em administração e em experiências envolvendo pesquisadores (grupos de pesquisa) e participação em eventos (MERRIAM, 1998; GEWANDSZNAJDER, 2001).

Os sujeitos deste estudo foram 24 gestores e 50 analistas de ambos os sexos, profissionais das áreas de planejamento, suporte operacional, administrativo, financeiro, programação, logística, expedição e gestão das unidades de negócios da cooperativa estudada no Norte e Nordeste do Brasil. Os gestores da cooperativa foram escolhidos por conhecerem o fluxo do conhecimento na organização e instituírem os modelos e as políticas de sua gestão.

$\mathrm{O}$ instrumento utilizado para a coleta dos dados foi o proposto por Bukowitz e Williams (2002) e traduzido para o Brasil, tendo sido publicado em 2002. O instrumento é composto por 140 questões, divididas em sete seções (processo tático: obtenha, utilize, aprenda e contribua, e processo estratégico: avalie, construa/mantenha e descarte), cada uma com 20 perguntas objetivas.

A coleta de dados realizou-se em um encontro presencial com os sujeitos da pesquisa ocorrido em junho/2014, quando houve o treinamento e a prática do preenchimento do instrumento de pesquisa como forma de garantir a confiabilidade (MERRIAM, 1998).

Para cada pergunta do questionário, Bukowitz e Williams (2002) adotam uma escala de respostas gradativas com intensidade crescente de 1 a 3 pontos, variando de fraco a forte, conforme o Figura 1.

\begin{tabular}{|l|c|}
\hline \multicolumn{2}{|l|}{ Pontos das Respostas Obtidas Pelo Questionário } \\
\hline Fortemente Descritiva (F) & 3 pontos \\
\hline Moderadamente Descritiva (M) & 2 pontos \\
\hline Fracamente Descritiva (Fr) & 1 ponto \\
\hline
\end{tabular}

Figura 1 - Escala de avaliação em ordem crescente Fonte: adaptado Bukowitz e Williams (2002).

De acordo com as autoras, a interpretação da pontuação dos resultados do Diagnóstico de Gestão do Conhecimento (DGC) acontece obedecendo aos critérios seguintes: quanto maior o percentual obtido nas respostas, melhor o desempenho de um passo específico no processo de Gestão do Conhecimento. Para calcular cada seção, utiliza-se do algoritmo apresentado na Figura 2. Os dados coletados foram tabulados em planilhas eletrônicas em que foi obtido o valor escalar médio para cada item avaliativo específico (Indicadores de GC - Quadro 2).

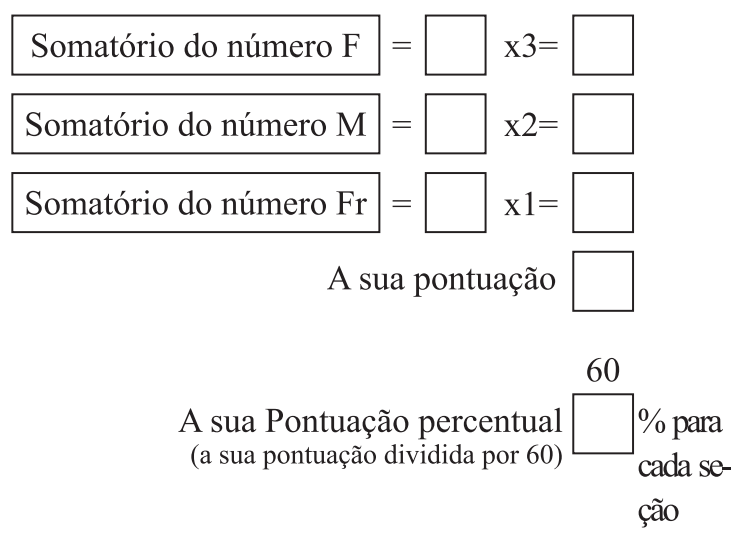

Figura 2 - Método para cálculo por Seção Fonte: Bukowitz e Wiliams (2002, p. 34).

Para a operacionalização do algoritmo, tem-se: os scores atribuídos ("Fs"=3, "Ms"=2 e "Fr"=1), soma-se o total de "Fs" e multiplica-se por três; o total de "Ms" multiplica-se por dois, e o total de "Fr" multiplicado por um. A pontuação máxima para cada seção é de 60 pontos (20 afirmações x 3); já a pontuação máxima possível geral (de todas as seções) é 420 pontos (60 pontos x 7 seções), conforme Figura 3 , sendo possível adaptar para leitura de acordo com o número de participantes da pesquisa. 


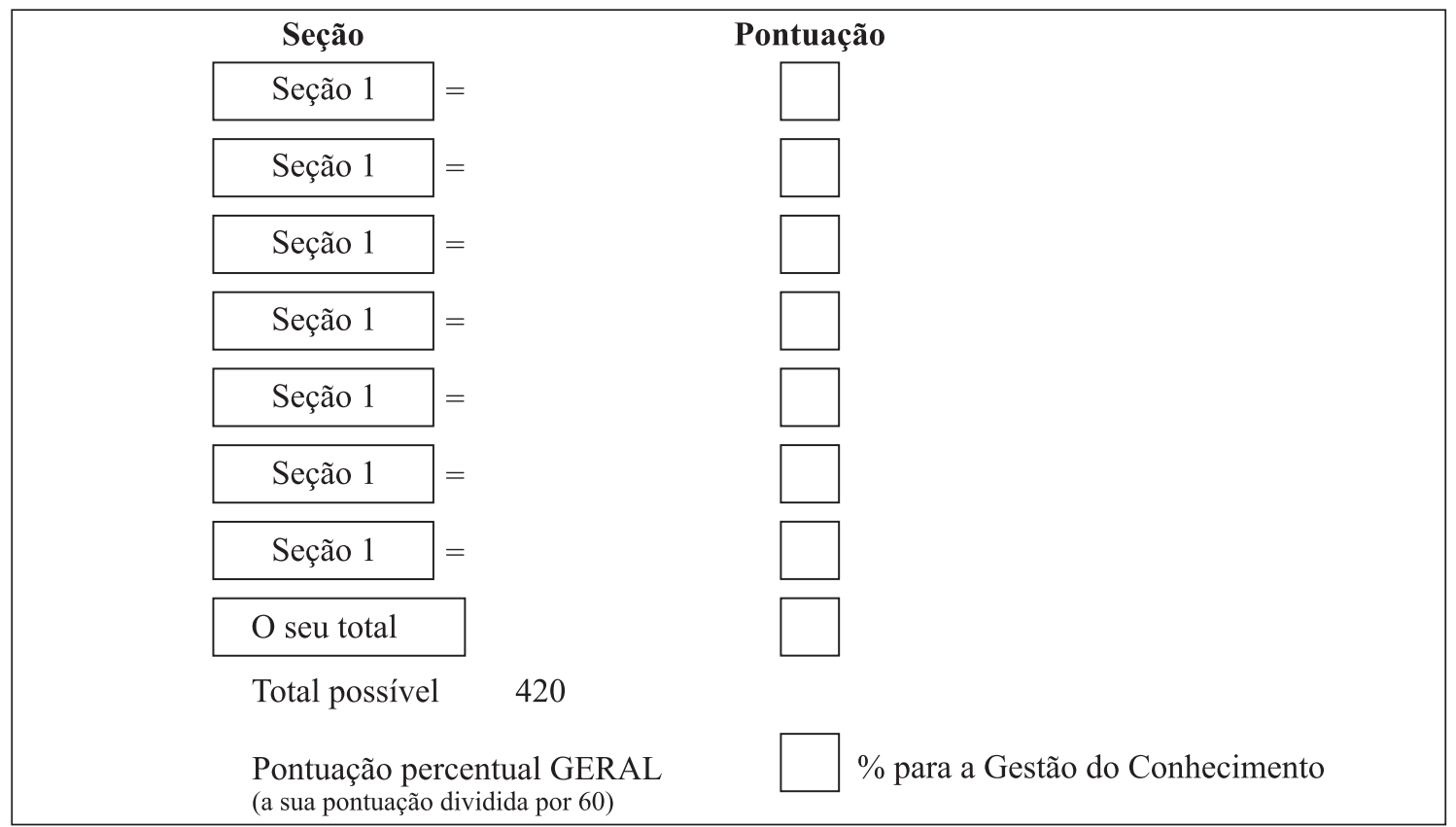

Figura 3 - Método para cálculo Geral

Fonte: Bukowitz e Wiliams (2002, p. 47).

Bukowitz e Williams (2002) adotam como "padrão aceitável" de gestão do conhecimento uma pontuação média, para cada seção de 30 a 70\%, pois são médias encontradas nos estudos que realizaram, e para todas as seções de $55 \%$.

Para aprofundamento da análise da Seção Avalie, utilizou-se dos indicadores de GC atribuídos às questões do instrumento de pesquisa conforme Brito, Oliveira e Castro (2012). A partir do instrumento de coleta de dados, (questionário testado e validado metodologicamente) estes autores desenvolveram uma escala de avaliação para o Domínio Cognitivo Seção Aprenda por meio de Itens Avaliativos Específicos (Indicadores de Gestão do Conhecimento) construídos, a partir da literatura pesquisada, e que foram transformados em uma escala de valores adequada para análise e mensuração. 


\begin{tabular}{|c|c|c|c|c|c|c|c|}
\hline $\begin{array}{l}\text { Indicadores de Ges- } \\
\text { tão do Conhecimento }\end{array}$ & Obtenha & Utilize & Aprenda & Contribua & Avalie & $\begin{array}{c}\text { Construa/ } \\
\text { Mante- } \\
\text { nha } \\
\end{array}$ & Descarte \\
\hline $\begin{array}{l}\text { Processos de gestão do } \\
\text { conhecimento/ } \\
\text { contextualização/ } \\
\text { Política de Gestão do } \\
\text { conhecimento }\end{array}$ & - & - & $\begin{array}{c}1,10,12 \\
15\end{array}$ & $\begin{array}{l}2,11,12 \\
15,16,18\end{array}$ & $\begin{array}{c}3,4,9, \\
10,13, \\
14,15, \\
17,18, \\
20\end{array}$ & $\begin{array}{c}1,6,7,8 \\
16,17 \\
18,19\end{array}$ & $\begin{array}{l}2,10,13 \\
16,17,19\end{array}$ \\
\hline $\begin{array}{l}\text { Compartilhamento } \\
\text { do conhecimento/ } \\
\text { Comunicação/ } \\
\text { Relacionamento }\end{array}$ & $\begin{array}{c}1,2 \\
4,8,9\end{array}$ & $\begin{array}{c}1,5 \\
8,15 \\
18,19\end{array}$ & 1,19 & $\begin{array}{c}5,7,8,10 \\
11,12 \\
13,14 \\
16,17 \\
18,19,20\end{array}$ & $2,8,12$ & $\begin{array}{c}1,2,3 \\
4,19\end{array}$ & $2,4,9,19$ \\
\hline $\begin{array}{l}\text { Papéis de gestão do } \\
\text { conhecimento }\end{array}$ & $\begin{array}{c}3,6,10 \\
12 \\
13,18\end{array}$ & - & - & $1,9,16$ & - & 3,1 & 6 \\
\hline $\begin{array}{l}\text { Estrutura física e } \\
\text { organizacional }\end{array}$ & - & $\begin{array}{c}2,3 \\
11,13 \\
17\end{array}$ & - & $4,8,10$ & - & - & - \\
\hline $\begin{array}{l}\text { Meios eletrônicos e } \\
\text { tecnologias } \\
\text { da informação }\end{array}$ & $\begin{array}{c}5,7,11 \\
14, \\
16,17\end{array}$ & - & - & 4,1 & - & $\begin{array}{c}2,5,11 \\
13,17\end{array}$ & - \\
\hline $\begin{array}{l}\text { Documentação, } \\
\text { resultados, } \\
\text { Mensuração / } \\
\text { protocolos e regras }\end{array}$ & $2,19,20$ & 14 & - & - & $\begin{array}{c}3,4,5 \\
6,7,8 \\
11,12 \\
20\end{array}$ & - & - \\
\hline Processo decisório & - & 4 & $\begin{array}{c}2,6,8 \\
17,18 \\
20\end{array}$ & - & 13 & $\begin{array}{c}1,3,8 \\
13,14,15\end{array}$ & $\begin{array}{c}1,3,8 \\
13,14,15\end{array}$ \\
\hline $\begin{array}{l}\text { Simulação/ jogos/ } \\
\text { inovação/ } \\
\text { Resolução de } \\
\text { problemas }\end{array}$ & - & $\begin{array}{l}6,8,9 \\
12,20\end{array}$ & $\begin{array}{c}4,11,13 \\
16,20\end{array}$ & - & - & 2,14 & 7,18 \\
\hline Parceria/ resultados & - & $\begin{array}{c}7, \\
10,16\end{array}$ & 3,9 & $\begin{array}{c}3,5,6 \\
7,14,15 \\
16,17\end{array}$ & - & $\begin{array}{c}2,8,12 \\
15,18,20\end{array}$ & $11,12,19$ \\
\hline $\begin{array}{l}\text { Avaliação do } \\
\text { conhecimento }\end{array}$ & - & - & $\begin{array}{c}5,7,9 \\
14\end{array}$ & - & - & - & $5,13,16$ \\
\hline $\begin{array}{l}\text { Reconhecimento e } \\
\text { valorização } \\
\text { da gestão do } \\
\text { conhecimento }\end{array}$ & - & - & - & 3,17 & $\begin{array}{c}1, \\
16,19\end{array}$ & $9,10,20$ & 10,2 \\
\hline
\end{tabular}

Quadro 2 - Distribuição dos itens da pesquisa avaliativa segundo os indicadores de Gestão do Conhecimento

Fonte: adaptado de Brito, Oliveira e Castro (2012).

Portanto, a pontuação obtida na pesquisa avaliativa foi padronizada para uma escala Likert de variação de 0 a 6 pontos (LIKERT, 1932), em que o valor 0 corresponde à resposta "fracamente 
descritiva"; o valor 3 pontos corresponde à resposta "moderadamente descritiva"; e, o valor 6 pontos como "fortemente descritiva". O intuito da transformação das pontuações foi facilitar a análise dos valores médios finais obtidos pela pesquisa em categorias de estudo mais adequadas para avaliação, conforme Tabela 1 .
Trata-se de uma sociedade cujo capital é formado pelos associados e tem a finalidade de somar esforços para atingir objetivos comuns que beneficiem a todos. Possui mais de 24 mil médicos credenciados e mantém filiação com diversas empresas criadas para oferecer ações e serviços de suporte e melhor atendimen-

Tabela 1 - Escala padronizada de avaliação em ordem crescente por categorização

\begin{tabular}{c|l|lc}
\hline \multicolumn{1}{c|}{ Escala de valores } & \multicolumn{1}{c|}{ Descrição } & \multicolumn{1}{c}{ Categorização } \\
\hline $0 \mid-2$ & Maior ou igual a 0 e menor que 2 & FRACO \\
\hline $2 \mid-4$ & Maior ou igual a 2 e menor que 4 & MODERADO \\
\hline $4 \mid-6$ & Maior ou igual a 4 e menor ou igual a 6 & FORTE \\
\hline 0 & 2 & 3 & 4 \\
\hline
\end{tabular}

Fonte: (BRITO; OLIVEIRA; CASTRO, 2012).

Segundo Godoy (1995, p. 26):

Ainda que os estudos de caso sejam, em essência, pesquisa de caráter qualitativo, podem comportar dados quantitativos para aclarar algum aspecto da questão investigada. É importante ressaltar que, quando há análise quantitativa, geralmente o tratamento estatístico não é sofisticado.

Em seguida, tem-se a apresentação e discussão dos resultados por meio de uma descrição rica e densa que é a maior estratégia para garantir a validade externa da pesquisa (MERRIAM, 1998).

\section{APRESENTAÇÃO E DISCUSSÃO DOS RESULTADOS}

\subsection{CARACTERIZAÇÃO DA ORGANI- ZAÇÃO E DOS PESQUISADOS}

A organização estudada é uma das maiores cooperativas médicas do Brasil, a maior do Norte e Nordeste, e possui mais de 37 anos de existência. A cooperativa abrange $85 \%$ dos municípios das regiões Norte e Nordeste oferecendo serviços em saúde de baixa, média e alta complexidade. to a seus clientes, ampliando-se os resultados e oportunizando novos negócios. Trata-se de uma rede de serviços na qual estão incluídos 18 hospitais próprios, 1.098 leitos hospitalares, 396 hospitais e 18.920 leitos referenciados. A rede da cooperativa no Norte e Nordeste produz 10,8 milhões de consultas e 25,9 milhões de exames laboratoriais por ano e, além disso, são gerados mais de 9 mil empregos diretos e 58 mil indiretos (SANTOS, 2014).

Com relação aos pesquisados, observou-se que a maior parte era formada por homens, casados, com faixa etária entre 26 e 45 anos, que possuíam escolaridade igual ou superior ao nível de graduação - 53,1\% do total. $36,7 \%$ dos pesquisados possuíam curso de especialização, $8,2 \%$ mestrado e apenas $2,0 \%$ eram possuidores do título de doutor.

\subsection{DIAGNÓSTICO DA GESTÃO DO CONHECIMENTO}

De acordo com o modelo proposto por Bukowitz e Williams (2002), é possível diagnosticar a Gestão do Conhecimento analisando-se em quais seções/fases a instituição se encontra mais ou menos focada. 
Tabela 2 - Resultado geral das pontuações e percentuais obtidos por seção

\begin{tabular}{|c|c|c|}
\hline Seção & Percentual da seção (\%)* & Medida de desempenho \\
\hline \multicolumn{3}{|c|}{ PROCESSO TÁTICO } \\
\hline Obtenha & 65,2 & Melhor desempenho \\
\hline Utilize & 60,7 & \\
\hline Aprenda & 65,0 & \\
\hline Contribua & 63,3 & \\
\hline \multicolumn{3}{|c|}{ PROCESSO ESTRATÉGICO } \\
\hline Avalie & 53,3 & Desempenho mais fraco \\
\hline Construa / Mantenha & 63,3 & \\
\hline Descarte & 65,0 & \\
\hline Total & 60,2 & \\
\hline
\end{tabular}

Fonte: dados da pesquisa (2014).

(*) Este valor representa o somatório das escolhas dos respondentes em todos os questionários multiplicados pelos scores ("F"=3), ("M"=2) e ("Fr"=1), dividido por 60 pontos, sendo que o total representa o somatório dos pontos obtidos nas seções dividido por 420 pontos.

Com base na pontuação obtida em cada etapa (seção), pode-se perceber que a instituição está no padrão aceitável de Gestão do Conhecimento por seção, pois os valores de cada seção precisam estar entre 30 a $70 \%$, conforme o modelo das autoras Bukowitz e Williams (2002), sendo o desempenho aceitável para cada uma das sete seções (quatro seções no processo tático e três seções no estratégico). Também, para todas as seções, o resultado encontrado foi aceitável, sendo de $60,2 \%$, acima do valor $55 \%$ segundo as autoras, revelando que, de forma geral, foi aceitável o padrão de Gestão do Conhecimento da instituição.
A mesma tendência ocorreu em cada processo (tático e estratégico) que alcançou respectivamente $63,55 \%$ e $60,53 \%$. O processo tático refere-se à forma como as pessoas tratam diariamente o conhecimento e o utilizam como ferramenta para atender as rotinas empresariais. Já o processo estratégico revela o alinhamento entre as estratégias do conhecimento com as estratégias organizacionais, conforme os resultados descritos no Quadro 3, aferidos por meio da intensidade das repostas dos sujeitos na fase de coleta dos dados. 


\begin{tabular}{|c|c|c|c|}
\hline Processo & Seção & $\%$ & Resultado dos objetivos específicos \\
\hline \multirow{4}{*}{ Tático } & Obtenha & 65,2 & $\begin{array}{l}\text { A obtenção do conhecimento foi o ponto mais forte da instituição } \\
\text { estudada no cenário tático. Percebeu-se que o conhecimento é obtido de } \\
\text { forma moderada, por meio da competência das pessoas, na busca para } \\
\text { realização das tarefas; por meio de compartilhamento de informações, } \\
\text { conhecimento e experiências bem sucedidas; pesquisas em meios } \\
\text { eletrônicos, que são mantidos parcialmente atualizados, por meio } \\
\text { de indivíduos ou grupos que identificam, coletam e disseminam o } \\
\text { conhecimento na instituição, ainda que sejam necessárias medidas de } \\
\text { ação para melhorar as estruturas de processos a fim de compartilhar as } \\
\text { informações relativas ao conhecimento da instituição. }\end{array}$ \\
\hline & Utilize & 60,7 & $\begin{array}{l}\text { Verifica-se que o conhecimento é utilizado na cooperativa por meio } \\
\text { da construção de parcerias para favorecer os beneficiários e, de forma } \\
\text { moderada, com promoção de espaços para reflexão e compartilhamento } \\
\text { do conhecimento; no processo decisório, para agregar valor ao mercado } \\
\text { ou quando as pessoas têm uma ideia ou opinião a oferecer a fim de } \\
\text { melhorar o trabalho. Dessa forma, faz-se necessário que a instituição } \\
\text { aprimore sua capacidade de utilizar o conhecimento por meio de } \\
\text { simulação / jogos / inovação, com o intuito de ampliar sua capacidade } \\
\text { de documentação e mensuração. }\end{array}$ \\
\hline & Aprenda & 65,0 & $\begin{array}{l}\text { Constatou-se que a instituição empreende ações voltadas para o } \\
\text { aprendizado individual e organizacional, reconhecendo o valor da } \\
\text { educação aplicada, identificando as formas de aprendizagem no } \\
\text { cotidiano e integrando os mecanismos de reflexão no hábito do } \\
\text { trabalho. Ainda se observou, de forma moderada, a avaliação do } \\
\text { conhecimento construído por meio da interação entre as pessoas, o } \\
\text { que pode prejudicar o entendimento de como acontecem os fatos na } \\
\text { instituição, e a aprendizagem sobre como resolver os problemas do dia } \\
\text { a dia organizacional. }\end{array}$ \\
\hline & Contribua & 63,3 & $\begin{array}{l}\text { Observou-se que a instituição aprende de forma moderada, isto é, } \\
\text { a capacidade de conseguir que as pessoas contribuam para a base } \\
\text { de conhecimento organizacional com o que aprenderam. São fracas } \\
\text { a integração e a legitimação dos processos que visam contribuir } \\
\text { com o conhecimento para a organização. Também são fracos o } \\
\text { reconhecimento e a valorização das pessoas que contribuem com o } \\
\text { conhecimento para a organização. Também se constatou que é pífia } \\
\text { a utilização de tecnologias da informação capazes de contribuir com } \\
\text { seu armazenamento e direcionamento. As pessoas não são capazes de } \\
\text { influenciar e contribuir por meio da socialização de ideias e expertises } \\
\text { com foco na informação, que agregue à missão da instituição. Faz-se } \\
\text { necessário aprimorar os meios eletrônicos para que sejam capazes de } \\
\text { contribuir com o armazenamento e o direcionamento da informação, } \\
\text { incentivando e facilitando a participação das pessoas de múltiplas } \\
\text { comunidades, compartilhando seu conhecimento; integrar e legitimar } \\
\text { processos de expansão do conhecimento, e reconhecer e valorizar as } \\
\text { pessoas que contribuem para o conhecimento da instituição. }\end{array}$ \\
\hline
\end{tabular}




\begin{tabular}{|c|c|c|c|}
\hline \multirow{3}{*}{ Estratégico } & Avalie & 53,3 & $\begin{array}{l}\text { Identificou-se que o conhecimento e as informações existentes na } \\
\text { instituição estudada não são avaliados de forma sistêmica e rotineira, o } \\
\text { que pode acarretar impedimento à mudança e às repostas exigidas pelo } \\
\text { mercado, uma vez que não se tem, ou tem-se pouca informação sobre } \\
\text { o que realmente é importante para a instituição alcançar sua missão. } \\
\text { Faz-se necessário adotar medidas no sentido de mensurar e descrever } \\
\text { os fluxos do conhecimento de modo a promover o entendimento sobre } \\
\text { o funcionamento da empresa e seus resultados. }\end{array}$ \\
\hline & $\begin{array}{l}\text { Construa/ } \\
\text { Mantenha }\end{array}$ & 63,3 & $\begin{array}{l}\text { Observou-se que o conhecimento se constrói/mantém moderadamente, } \\
\text { quando a cooperativa utiliza tecnologias compartilhadas como fonte } \\
\text { confiável de informação de que necessita para realizar o trabalho; } \\
\text { quando a instituição percebe que não é apropriado compartilhar o } \\
\text { conhecimento externa e internamente o comunica para que todos } \\
\text { se beneficiem. Por outro lado, é importante adotar medidas de ação } \\
\text { para promover indivíduos ou grupos capazes de liderar o esforço de } \\
\text { Gestão do Conhecimento na instituição. Além disso, construir redes de } \\
\text { relacionamento com outras organizações e clientes para compartilhar } \\
\text { tecnologias e ideias e buscar alinhar os valores formais e informais } \\
\text { entre as pessoas e as organizações. }\end{array}$ \\
\hline & Descarte & 65,0 & $\begin{array}{l}\text { Verifica-se que o conhecimento não é descartado moderadamente } \\
\text { na cooperativa e que moderadamente se busca rever as práticas de } \\
\text { valorização de pessoal, pois pouco se leva em conta o impacto de deixar } \\
\text { sair pessoas com capacidade de contribuir com lealdade e compromisso } \\
\text { quando se reflete pouco sobre a possibilidade de não descartar } \\
\text { o conhecimento construído; quando as pessoas moderadamente } \\
\text { participam de grupos de discussão sobre o ramo do negócio da } \\
\text { instituição, pois podem estar deixando de adquirir conhecimento que } \\
\text { agregue valor para a instituição. }\end{array}$ \\
\hline
\end{tabular}

Quadro 3 - Resultado dos objetivos específicos

Fonte: dados da pesquisa (2014).

A partir da análise dos resultados, também foi possível perceber que existem pessoas na instituição que contribuem ajudando as outras a se expressarem, ou seja, a explicitar o conhecimento tácito, apesar de não serem formalmente designadas, já que se verificou que a instituição de forma fraca lança indivíduos ou grupos específicos capazes de liderar os esforços de GC.

De forma recorrente, em todas as seções do DGC, com exceção da seção utilize, percebeu-se que o indicador "Documentação, Resultados, Mensuração / Protocolos e Regras" apresentou intensidade moderada, demonstrando que a instituição moderadamente utilizava fatos sólidos, números, informações não mensuráveis, resultados de jogos e simulações para tomar decisões ou entender melhor o que estava tentando gerir e seus resultados. Isso pode ter impactado diretamente o processo decisório e no planejamento organizacional, demonstrando-se que a instituição entendia parcialmente como os fatos aconteciam, pouco aprendia para resolver as situações do dia a dia e produzia capacidade reduzida de aprendizagem para prever ações futuras a partir das experiências do passado.

Além disso, a cooperativa enxergava com intensidade fraca a moderada a parceria com os concorrentes e beneficiários como condição para produzir ganhos mútuos; portanto, não fazia uso das parcerias para produzir aprendizagem. A instituição também reconhecia que o conhecimento é um recurso capaz de gerar valor; porém, não era capaz de gerenciá-lo.

Os resultados ainda demonstraram que o conhecimento era descartado na cooperativa quando ela não buscava rever suas práticas de 
valorização de pessoal, pois pouco levava em conta o impacto de deixar ir embora pessoas com capacidades de contribuição, lealdade e compromisso; não refletia sobre como o conhecimento construído pode ser utilizado de outras maneiras antes de descartá-lo, o que teria causado perda de capital intelectual.

Existem sinalizações de que a organização estudada administrava seus conflitos cognitivos e possuía uma boa condição logística e tecnológica para consolidar a Gestão do Conhecimento (KIMMERLE; CRESS; HELD, 2010; SOMALINGAN; SHANTHAKUMARI, 2013). O compartilhamento do conhecimento pela internalização, socialização, externalização e combinação (NONAKA; TAKEUCHI, 1997), acontecia de maneira, ainda, informal. A proximidade social e conceitual, a confiança, os valores culturais, e os fatores estruturais, como a administração, o financiamento e a colaboração parecem ser favoráveis, o que facilitaria a interpretação e a construção do conhecimento (HAUTALA, 2011).

Os resultados gerais que indicam como ponto forte a obtenção do conhecimento e como ponto mais fraco a avaliação estão em concordância com os resultados obtidos em outras pesquisas (COSTA; VASCONCELOS; CANDIDO, 2009; DIHL; HOLANDA; FRANCISCO, 2010; CASTRO, 2011; BRITO; OLIVEIRA; CASTRO, 2012; BOLSON, 2012; GALVÃO, 2012; GONZAGA, 2014; SANTOS, 2014; BRITO; OLIVEIRA, CASTRO, 2016) que utilizaram o mesmo modelo teórico de Bukowitz e Williams (2002). Com relação à carência relacionada na seção avaliação (nível estratégico), o fenômeno também foi observado no estudo de Gonçalves, Lima e Costa (2009) em uma organização cooperativa rural utilizando outra metodologia.

Em um estudo do estado da arte da Gestão do Conhecimento em organizações públicas, incluindo-se as da área de saúde, como centros de pesquisa clínica - unidades vinculadas a hospitais universitários e a outras organizações de saúde) e organizações adesas ao Programa Nacional de Gestão Pública e Desburocrati- zação - (GesPública), e na Agência Nacional de Saúde Suplementar (ANS), também se percebeu que essas várias organizações de saúde ainda não adentravam no nível estratégico da gestão do conhecimento (CASTRO; BRITO, 2016). A mesma tendência ocorreu em outros estudos da área (BERNARDO et al., 2016).

Segundo Bukowitz e Williams (2002), a fragilidade apresentada na seção avalie do diagnóstico pode acarretar à cooperativa médica estudada um impedimento à mudança e as respostas exigidas pelo mercado, uma vez que há um comprometimento das informações sobre o que realmente é importante para a organização se manter viável no futuro, frente as suas estratégias.

De acordo com Silva e Binotto (2013) ,para que ocorra a criação do conhecimento no segmento de cooperativa, ainda são necessários avanços significativos, como escolher um processo de aprendizagem voltado para o conhecimento organizacional, que ocorra de forma coletiva, a fim de ampliar a visão de todo grupo e não somente para a informação que regularize os processos produtivos para atender às normativas do setor.

Os resultados indicaram que a organização está com os processos de Gestão do Conhecimento, com índices acima da média, ou seja, $60,2 \%$, entretanto que precisa melhorar o processo de avaliação. Inúmeros fatores podem ocasionar a dificuldade das organizações em avaliar a Gestão do Conhecimento tais como: a existência ou não de uma Política de Gestão do Conhecimento formal e conhecida por todos envolvidos no processo, a definição dos papéis no processo da gestão do conhecimento, o processo decisório, a estrutura física, logística e organizacional, a comunicação organizacional, os meios eletrônicos e a tecnologia da informação, a forma como é feita a documentação, a apresentação dos resultados, a mensuração das metas, os protocolos, as regras e como se dá o trabalho dos especialistas do conhecimento na organização, que são os que filtram os conhecimentos realmente necessários para alavancar a criação no conhecimento em seu interior. 


\section{CONSIDERAÇÕES PROVISÓRIAS}

Considerando o problema de partida e o objetivo geral proposto por este trabalho, a análise dos dados indica que na percepção dos gestores pesquisados, a organização encontra-se em um estágio moderado no processo de Gestão do Conhecimento; ou seja, já tem implantada formalmente a Gestão do Conhecimento no nível tático e no nível estratégico.

Com relação aos objetivos específicos, o desempenho médio da pesquisa ultrapassou o previsto como aceitável pelas idealizadoras do instrumento, no nível tático - obter, utilizar, aprender e contribuir. A seção que teve a menor pontuação no processo tático foi a seção utilize. Constatou-se, ainda, estágio superior ao previsto aceitável pelas idealizadoras do instrumento para o nível estratégico - avaliar, construir e descartar. A seção que obteve a menor avaliação no processo estratégico foi a seção avalie.

Partindo-se do princípio de que o principal recurso organizacional é o conhecimento, observa-se, pelos resultados, que a organização possui potencial para a criação do conhecimento, pois nenhuma das seções obteve resultado fora do range proposto pelas autoras $(30 \%$ a $70 \%$ ). Com uma média geral de $60,2 \%$ alcançou limite acima do proposto de $55 \%$.

Dessa forma, conclui-se que a organização objeto do estudo, apesar de ser uma referência no cooperativismo médico regional e nacional e estando inserida em um mercado cada vez mais competitivo, poderia ter apresentado um resultado mais expressivo, no que concerne à utilização (processo tático) e avaliação (processo estratégico) do conhecimento.

Respectivamente, implica dizer que, primeiro: (utilização), os membros da organização teriam alguma dificuldade em combinar a informação de maneira que satisfizessem seus clientes com qualidade, pois a inovação constante (marca do atual ambiente organizacional) força as pessoas a saírem de seus limites funcionais, operativos e de processos para procurar ideias escondidas, que não seriam consideradas, para se oferecer um serviço em constante nível de qualidade.
Por outro lado, no nível da organização, o impacto desse resultado se dá no ambiente de trabalho, no qual a organização pouco está encorajando a criatividade, a experimentação e a receptividade, refletindo diretamente, por exemplo, na imagem da organização frente ao mercado (clientes), referente ao conhecimento que seus médicos cooperados ofertam à população.

Como segundo aspecto, (avaliação) remete ao fato de que a organização ainda não atravessou totalmente o limiar entre os dois fluxos do processo geral de gestão do conhecimento (tácito para estratégico) que se dá por meio das respostas às mudanças no ambiente macroeconômico e manifesta-se por alterações de direção estratégica e variações na velocidade e intensidade das respostas organizacionais ao mercado. Esse processo estratégico contínuo é responsabilidade dos gestores em nível estratégico da organização, e impacta diretamente no futuro da organização, no compartilhamento com todos os stakeholders de indicadores para uma avaliação rigorosa e contínua de todos os momentos do processo de gestão do conhecimento organizacional visando à excelência de sua missão, visão de futuro, valores, negócio e estratégias.

Apesar disso, as limitações desta pesquisa residem na indisponibilidade dos sujeitos da pesquisa (gestores e analistas) de participarem de entrevistas, para se analisar, a partir da subjetividade constituída pela atividade, e na atividade, quais os impedimentos para o desenvolvimento do processo estratégico (seção avalie) da GC, que impossibilitariam a manutenção da organização no futuro.

Não se pretendia com esta pesquisa esgotar todas as possibilidades sobre o tema estudado (Gestão do Conhecimento), o qual constitui em um modelo teórico em evolução.

Portanto, trabalhos futuros poderão originar novas abordagens como: comparativasobre o estágio da Gestão do Conhecimento na percepção de gestores e analistas obtidos a partir de pesquisas junto a outras cooperativas das regiões Norte e Nordeste; investigativa - sobre de que forma a Gestão como o Conhecimento contribui para o posicionamento da cooperativa 
no âmbito nacional frente a seus clientes; de verificação - quanto ao nível de consolidação de uma cultura de gestão do conhecimento na cooperativa; e, ainda, estudos críticos: que abordem como a Gestão do Conhecimento pode evoluir para contribuir na solução dos problemas da sociedade à luz da filosofia, sociologia, educação, entre outras, suplantando o uso do conhecimento do nível organizacional para o nível social, superando os estudos funcionalistas em favor de um olhar da compreensão holista.

\section{KNOWLEDGE MANAGEMENT IN A MEDICAL COOPERATIVE IN NORTHERN-NORTHEASTERN BRAZIL}

\begin{abstract}
The objective of the study was to analyze the Knowledge Management (CG) stage in the perception of managers and analysts of a medical cooperative in Northern-Northeastern Brazil. The theoretical model used was that of Bukowitz and Williams (2002) with definition of variables in Brito, Oliveira and Castro (2012). This is a qualitative research, with a case study mode of investigation and a descriptive approach. The subjects of the research were the 24 managers and 50 analysts of the cooperative. The data was collected through questionnaires and treated according to the algorithm of the model in electronic spreadsheets. The results indicate higher values than those proposed by Bukowtiz and Williams (2002), demonstrating the implantation of the $\mathrm{CG}$ at both the tactical and strategic levels in the organization under analysis. As such, it is possible to observe that the organization provides facilitating conditions for the creation, recognition of tacit knowledge, dissemination and incorporation of knowledge into technologies and products. However, the organization has not been able to consolidate the evaluation phase, which is the moment in which one defines the knowledge necessary for its maintenance in the future.
\end{abstract}

Keywords: Knowledge Management. Medical Cooperatives. Northern and Northeastern Brazil.

\section{GESTIÓN DEL CONOCIMIENTO EN UNA COOPERATIVA MÉDICA DEL NORTE/NORESTE BRASILEÑO}

\section{RESUMEN}

El objetivo del estudio fue analizar el periodo de la Gestión del Conocimiento (GC) en la percepción de gestores y analistas de una cooperativa médica del norte-no/noreste brasileño. El modelo teórico utilizado fue lo de Bukowitz y Williams (2002) con definición de variables en Brito, Oliveira y Castro (2012). Se trató de una investigación cualitativa con un estudio de caso como modo de investigación y enfoque descriptivo. Los sujetos de la investigación fueron los 24 gestores y 50 analistas de la cooperativa bajo estudio. Los datos fueron colectados por medio de cuestionarios y tratados de acuerdo con el algoritmo del modelo en hojas de cálculo electrónicas. Los resultados indicaron valores superiores a los propuestos por Bukowitz y Williams (2002), lo que demuestra la implementación de la GC en los niveles táctico y estratégico en la organización bajo análisis. Así, es posible observar que la organización propicia condiciones que facilitan la creación, el reconocimiento del conocimiento tácito, la diseminación y la incorporación del conocimiento a las tecnologías y productos; sin embargo, la organización todavía no ha logrado consolidar la fase de evaluación, que es el momento en que se define el conocimiento necesario para su manutención en el futuro.

Palabras-clave: Gestión del Conocimiento. Cooperativas Médicas. Norte/Noreste Brasileño. 


\section{LA GESTION DES CONNAISSANCES DANS UNE COOPERATIVE MEDICALE DU NORD/NORD-EST BRESILIEN}

\section{RESUME}

L'objetif de l'étude a été d'analyser le stage de la gestion des connaissances (GC) dans la perception des gérants et analystes d'une coopérative médicale du nord/nord-est brésilien. Le modèle théorique utilisé fut celui de Bukowitz et Williams (2002) avec la définition de variables de Brito, Oliveira et Castro (2012). La recherche a été qualitative, avec une étude de cas comme méthode d'enquête et une approche descriptive. Les sujets de l'investigation ont été les 24 gérants et 50 analystes de la coopérative sous étude. Les données ont été récoltées à travers des questionnaires et traités conformément à l'algorithme du modèle dans des feuilles de calcul éléctroniques. Les résultats ont indiqué des valeurs supérieures à celles proposées par Bukowitz et Williams (2002), ce qui démontrent l'implémentation de de la GC au niveau tactique et au niveau stratégique dans l'organisation sous étude. Ainsi, il est possible observer que l'organisation établit des conditions qui facilitent la création, la reconnaissance des savoirs tacites, la dissémination et l'incorporation des connaissances aux technologies et aux produits ; toutefois, l'organisation n'a pas réussi à consolider la phase d'évaluation, le moment quand les connaissances nécessaires à sa manutention sont définies.

Mots-clés: Gestion des Connaissances. Coopératives Médicales. Nord/Nord-est Brésilien.

\section{REFERÊNCIAS}

BERNARDO, F. D. et al. A gestão do conhecimento na unidade descentralizada do serviço de auditoria do SUS em Santa Catarina. Revista Gestão \& Planejamento, Salvador, v. 17, n. 3, p. 463-479, set./dez. 2016.
BOLSON, S. Gestão do conhecimento: estudo em uma instituição de ensino superior tecnológico. 2012. 101 f. Dissertação (Mestrado Profissional em Administração) - Universidade Potiguar, Natal, 2012.

BOXENBAUM, E.; ROULEAU, L. New knowledge products as bricolage: metaphors and scripts in organizational theory. Academy of Managament Review, New York, v. 36, n. 2, p. 272-296, 2011.

BRITO, L. M. P. Gestão de competências, gestão do conhecimento e organizações de aprendizagem: instrumentos de apropriação pelo capital do saber do trabalhador. Fortaleza: Imprensa Universitária, 2005.

; OLIVEIRA, P. W. S. de; CASTRO, A.

B. C. de. Gestão do conhecimento numa instituição pública de assistência técnica e extensão rural do Nordeste do Brasil. Revista de Administração Pública, Rio de Janeiro, v. 46, n. 5, p. 1341-1366, set./out. 2012.

; OLIVEIRA, J. A. de; CASTRO, A. B. C. de. Gestão do conhecimento: um estudo em um Hospital Federal Universitário do Nordeste do Brasil. Revista Gestão \& Conhecimento, Curitiba, v. 10, n. 2, p. 1-29, jul./dez. 2016.

BUKOWITZ, W. R.; WILLIAMS, R. L. Manual de gestão do conhecimento. São Paulo: Bookman, 2002.

CASTRO, A. B. C. de. Gestão do conhecimento: Um estudo em uma Instituição pública de assistência e extensão rural. 2011. Dissertação (Mestrado em Administração) - Universidade Potiguar, Natal, 2011.

.; BRITO, L. M. P. Como o IFRN/Campus são Paulo do Potengi aprende? In: CASTRO, A. B. C. de et al. (Org.). Práticas educativas em movimento: experiências formativas de ensino, pesquisa, extensão e gestão no Campus São Paulo do Potengi do IFRN. Natal: IFRN Editora, 2016. 
CHON, C. W.; BESHARATI, J. Challenges of knowledge sharing in the petrochemical industry. Knowledge Management \& E-Learning, China, v. 6, n. 2, p. 171-187, June 2014.

COSTA, I.; VASCONCELOS, A. C. F. de; CANDIDO, G. A. Diagnóstico de gestão do conhecimento como mecanismo para criação de valor: um estudo exploratório no SEBRAE-PB. Revista Gestão Industrial, Paraná, v. 5, n. 2, p. 80-98, 2009.

DAVENPORT, T.; PRUSAK, L. Conhecimento empresarial: como as organizações gerenciam o seu capital intelectual. Rio de Janeiro: Elsevier, 1998.

DIHL, W.; HOLANDA, L. M. C. de; FRANCISCO, A. C. de. A utilização do método de diagnóstico para a gestão do conhecimento: estudo de caso numa empresa incubada (ALFA). Revista eletrônica FAFIT/FACIC, São Paulo, v. 1, n. 1, p. 30-43, jan./jun. 2010.

GALVÃO, A. P. Gestão do conhecimento em empresa internacional de energia. 2012. 133 f. Dissertação (Mestrado Profissional em Administração) - Universidade Potiguar, Natal, 2012.

GEWANDSZNAJDER, F. Uma visão geral do método nas ciências sociais. In: ALVES-MAZOTTI, A. J.; GEWANDSZNAJDER, F. O método nas ciências naturais e sociais: pesquisa quantitativa e qualitativa. 2. ed. São Paulo: Pioneira Thomson Learning, 2001.

GODOY, A. S. Pesquisa qualitativa: tipos fundamentais. Revista de Administração de Empresas, São Paulo, v. 35, n. 3, p. 20-29, 1995.

GONÇALVES, L. C.; LIMA, E. P.; COSTA, S. E. G. Um estudo sobre a adoção de práticas de gestão do conhecimento em organizações cooperativas. Revista Produção, v. 19, n. 1, p. 163-189, jan./abr. 2009.
GONZAGA, A. Gestão do conhecimento em multinacional brasileira de energia. 2014. 128 f. Dissertação (Mestrado Profissional em Administração) - Universidade Potiguar, Natal, 2014.

GONZALEZ, R. V. D.; MARTINS, M. F. Mapping the organizational factors that support knowledge management in the Brazilian automotive industry. Journal of Knowledge Management, Sonning Common, v. 18, n. 1, p. 152176, 2014.

HAUTALA, J. International academic knowledge creation and ba. A case study from Finland. Knowledge Management Research \& Practice, United Kingdom, v. 9, p. 4-16, 2011.

JASIMUDDIN, S. M.; ZHANG, Z. Knowledge management strategy and organizational culture. J Oper Res Soc. United Kingdom,v. 65, n. 10, p. 1490-1500, 2014.

KIANTO, A. et al. The interaction of intellectual capital assets and knowledge management practices in organizational value creation. Journal of Intellectual Capital, United Kingdom, v. 15, n. 3, p. 362-375, 2014.

KIM, T. H. et al. Understanding the effect of knowledge management strategies on knowledge management performance: A contingency perspective. Information \& Management, Netherlands, v. 51, n. 4, p. 398-416, 2014.

KIMMERLE, J.; CRESS, U.; HELD, C. The interplay between individual and collective knowledge: technologies for organisational learning and knowledge building. Knowledge Management Research \& Practice, United Kingdom, v. 8, n. 1, p. 33-44, 2010.

LARA, B. et al. Knowledge management through two virtual communities of practice (Endobloc and Pneumobloc). Health Informatics Journal, United States, v. 23, n. 3, p. 170-180, 2017. 
LEE, H. Knowledge management enablers and process in hospital organizations. Osong Public Health and Research Perspectives, South Korea, v. 8, n. 1, p. 26-33, 2017.

LIKERT, R. A technique for the measurement of attitudes. Archives of psychology, New York, n. 140, p. 5-55, 1932.

MASSARO, M.; DUMAY, J.; GARLATTI, A. public sector knowledge management: a structured literature review. Journal of Knowledge Management, United Kingdom,v. 19, n. 3, p. 530-558, 2015.

MASSINGHAM, P. An evaluation of knowledge management tools: Part 1 - managing knowledge resources. Journal of Knowledge Management, Sonning Common, v. 18, n. 6, p. 1075-1100, 2014.

MEIHAMI, B.; MEIHAMI, H. Knowledge management a way to gain a competitive advantage in firms (evidence of manufacturing companies). International Letters of Social and Humanistic Sciences, v. 14, p. 80-91, 2014.

MERRIAM, S. Qualitative Research and Case Study Applications in Education. San Francisco: Jossey-Bass, 1998.

NOGUEIRA, R. A.; ODELIUS, C. C. Aprendizagem: evolução no contexto das teorias organizacionais. Perspectivas em Gestão \& Conhecimento, João Pessoa, v. 5, n. 1, p. 3-18, jan./jun. 2015.

NONAKA, I.; TAKEUCHI, H. Criação de conhecimento na empresa: como as empresas japonesas geram a dinâmica da informação. Rio de Janeiro: Campus, 1997.

PETROVA, M. et al. Between "the best way to deliver patient care" and "chaos and low clinical value": General Practitioners' and Practice Managers' views on data sharing. International Journal of Medical Informatics, Netherlands, v. 104, p. 74-83, 2017.
PINHEIRO, A. L. S. et al. Gestão da saúde: o uso dos sistemas de informação e o compartilhamento de conhecimento para a tomada de decisão. Texto \& Contexto Enfermagem, Florianópolis, v. 25, n. 3, p. 1-9, 2016.

PORTER, M. Competição estratégias competitivas essenciais. Rio de Janeiro: Campus, 1990.

SANTOS, R. V. Gestão do conhecimento em Cooperativa Médica do Norte/Nordeste Brasileiro. 2014. 97 f. Dissertação (Mestrado Profissional em Administração)- Universidade Potiguar, Natal, 2014.

SENGE, P. R. A quinta disciplina: arte e prática da organização que aprende. São Paulo: Best Seller, 2012.

SHEHZAD, R.; KHAN, M. N. A. Integrating knowledge management with business intelligence processes for enhanced organizational learning. International Journal of Software Engineering and Its Applications, South Korea, v. 7, n. 2, p. 83-92, 2013.

SILVA, I. F.; BINOTTO, E. O conhecimento e a aprendizagem organizacional no contexto de uma organização rural. Revista Perspectivas em Gestão \& Conhecimento, João Pessoa, v. 3, n. 1, p. 132-156, jan./jun. 2013.

SOMALINGAN, A.; SHANTHAKUMARI, R. Cross-cultural management: an empirical study on cultural identity and knowledge management of indian software engineers. International Journal of Information, Business and Management, v. 5, n. 2, p. 20-29, May 2013.

STEWART, T. A. Capital intelectual. 4. ed. Rio de Janeiro: Campus, 1998.

SVEIBY, K. E. A nova riqueza das organizações: gerenciando e avaliando patrimônios de conhecimento. Rio de Janeiro: Campus, 1998. 
TANG, H. A study of the effect of knowledge management on organizational culture and organizational effectiveness in medicine and health sciences. Eurasia Journal of Mathematics, Science and Technology Education, Turkey, v. 13, n. 6, p. 1831-1845, 2017.

TERRA, J. C. C. Gestão do conhecimento: o grande desafio empresarial. Rio de Janeiro: Negócio, 2005.

TZORTZAKI, A. M.; MIHIOTIS, A. A review of knowledge management theory and future directions. Knowledge and Process Management, Germany, v. 21, n. 1, p. 29-41, Jan./Mar. 2014.

VIRTANEN, I. In search for a theoretically firmer epistemological foundation for the relationship between tacit and explicit knowledge. The Electronic Journal of Knowledge Management, Sonning Common, v. 11, n. 2, p. 118126, 2013.

WAHYUNINGSIH, A.; ASTUTI, E. S.; MUSADIEQ, M. A. the effect of organizational learning on knowledge management, capability and performance of organization. Journal of Basic and Applied Scientific Research, Cairo, v. 3, n. 5, p. 159-169, 2013.

WONG, K. Y. et al. Knowledge management performance measurement: measures, approaches, trends and future directions. Information Development, United States, v. 31, n. 3, p. 239-257, 2015. 